\title{
ON THE CONCENTRATION AND EXTENSION OF CYLINDER MEASURES $\left({ }^{1}\right)$
}

\author{
BY \\ ALEJANDRO D. DE ACOSTA
}

\begin{abstract}
It is shown that with respect to certain set-theoretic operationsdirected decreasing or even arbitrary intersections of certain families of convex, balanced, weakly closed sets-cylinder measures behave almost as regular Borel measures do. A refinement is proved when the cylinder measure satisfies a scalar concentration condition. These results are applied to obtain stronger versions of Prohorov's theorem and an extension theorem for complete Hausdorff locally convex spaces generalizing a result of Dudley, Feldman, and Le Cam.
\end{abstract}

1. Introduction. The basic theorem of Prohorov ([9, p. 411], [1, p. 71], [3], [12], [13, Exposé No. 7]) states that a cylinder measure on a locally convex Hausdorff topological vector space $X$ extends to a regular Borel measure if and only if there exist compact sets of outer measure arbitrarily close to 1 . Given a compact set $K$, call a family $\mathscr{F}$ of cylinder sets containing $K$ fundamental if, for every cylinder set $C \supset K$, there is an $F \in \mathscr{F}$ such that $C \supset F \supset K$. Then the condition in Prohorov's theorem can be restated: For every $\varepsilon>0$, there exists a compact set $K$ and a fundamental family $\mathscr{F}$ of cylinder sets containing $K$, such that each $F \in \mathscr{F}$ has measure $\geqq 1-\varepsilon$. In fact, Prohorov's theorem is usually stated using the fundamental family $\left\{\pi^{-1}(\pi(K)): \pi\right.$ linear continuous on $X$, with finite-dimensional range $\}$. (In this form, a particular case of the theorem was proved by E. Mourier [8].)

It is of interest to know if a fundamental family can be replaced by a more general, less restrictive family of cylinder sets. If possible, this could allow a more flexible choice of the cylinder sets on which to focus attention in each particular space.

A significant result in this direction was obtained by L. Le Cam ([6], where it is announced without proof; also [7]). Particular cases, with a different formulation, were obtained by Getoor [5] and Fernique [4]. Roughly, Le Cam's theorem says that under certain conditions it is enough to prove that all "polyhedra" (that is, polars of finite subsets of the dual space) containing a compact (convex, balanced) set have measure close to 1 . (For a more precise statement, see Theorem 5.3.) It should be remarked that the family of "polyhedra" is not a fundamental family.

Received by the editors October 10, 1970.

AMS 1969 subject classifications. Primary 2846, 6008.

Key words and phrases. Cylinder measure, concentration up to $\varepsilon$, scalar concentration, Prohorov's extension theorem, extension of cylinder measures in complete spaces.

(1) This research was prepared with the partial support of the National Science Foundation Grant No. GP-22928. 
In the present work (Theorems 5.1 and 5.2), we show that in a natural geometric setting Prohorov's theorem can be strengthened by replacing a fundamental family with any of a wide class of directed decreasing families. Le Cam's theorem (Theorem 5.3) is obtained from Theorem 5.1.

An extension theorem for complete spaces (Theorem 5.4) generalizing in a certain sense a recent result of Dudley, Feldman and Le Cam [3] is proved in $§ 5$. Their result is included as Theorem 5.5.

The methods used in proving these results are developed in $\S 4$. Restricting attention to sets properly related to the linear topological structure makes possible the use of polarity arguments. It is shown that with respect to certain set-theoretic operations-directed decreasing intersections or even arbitrary intersections of certain families of "geometrically well-behaved" sets-cylinder measures behave almost as regular Borel measures do (Theorems 4.1 and 4.2). It turns out that our methods tie in naturally with the condition of scalar concentration of a cylinder measure, introduced by L. Schwartz [11]. Theorems 4.3, 4.4, and 5.2 involve a scalar concentration hypothesis.

As an initial more concrete application, Propositions 6.1 and 6.2 give extension conditions for cylinder measures on $l_{p}$ spaces.

Acknowledgement. I wish to thank Professor L. Le Cam for many stimulating conversations and for making his manuscript [7] available to me.

2. Preliminaries. All vector spaces considered will be real. Given a vector space $X$, its algebraic dual will be called $X^{*}$. The convex, balanced hull of a subset $A$ of $X$ will be denoted co $(A)$. The topological dual of a topological vector space $X$ will be called $X^{\prime}$. Given a (separating) dual system $(X, Y)$ of vector spaces, the weak topology on $X$ is denoted $\sigma(X, Y)$ and the Mackey topology on $X$ is denoted $\tau(X, Y)$ (similarly for $Y$ ). The polar of a subset $A$ of $X$ is the set $A^{0}=\{y \in Y:|\langle x, y\rangle| \leqq 1$ for all $x \in A\}$. We refer to Schaefer [10] for the linear space notions and results that we use.

The algebra of cylinder sets induced on $X$ by $Y$ is denoted $\mathscr{C}(X, Y)$. The $\sigma$ algebra induced on $X$ by a finite subset $F$ of $Y$ is denoted $\mathscr{S}(X, F)$. We use the standard definitions and basic facts about cylinder measures, which may be found in [1], [2], [3], [12], [13].

3. A geometric lemma. We will be interested in certain families of subsets of a topological vector space which have the property that their intersection is (roughly) preserved by weakly continuous linear maps.

Definition 3.1. Let $(X, Y)$ be a dual system of vector spaces. A family $\left\{K_{\alpha}\right\}_{\alpha \in I}$ of convex, balanced, $\sigma(X, Y)$-closed subsets of $X$ is said to be well-fitting if, for every real $\lambda>1$,

$$
K^{0} \subset \lambda\left(\operatorname{co} \bigcup_{\alpha \in I} K_{\alpha}^{0}\right), \quad \text { where } K=\bigcap_{\alpha \in I} K_{\alpha} \text {. }
$$


If $\left\{K_{\alpha}\right\}_{\alpha \in I}$ is directed downward by inclusion, then the well-fitting condition reduces to the following:

$$
\text { for every real } \lambda>1, \quad K^{0} \subset \lambda\left(\bigcup_{\alpha \in I} K_{\alpha}^{0}\right),
$$

for in this case $\bigcup_{\alpha \in I} K_{\alpha}^{0}$ is convex and balanced.

REMARK. Given any family $\left\{K_{\alpha}\right\}_{\alpha \in I}$ of convex, balanced, $\sigma(X, Y)$-closed sets, $K^{0}$ may be obtained from $\left\{K_{\alpha}^{0}\right\}_{\alpha \in I}$ through the formula

$$
K^{0}=\left[\bigcup_{\alpha \in I} K_{\alpha}^{0}\right]^{00}=\mathrm{cl}\left(\operatorname{co} \bigcup_{\alpha \in I} K_{\alpha}^{0}\right),
$$

the closure being taken with respect to any locally convex topology consistent with the duality.

Thus Definition 3.1 expresses a specially close relationship between $K^{0}$ and $\left\{K_{\alpha}^{0}\right\}_{\alpha \in I}$.

Examples of well-fitting families. (1) Let $K$ be a convex, balanced, $\sigma(X, Y)$ closed set. For every real $\lambda>1$, let $K_{\lambda}=\lambda K$. Then the family $\left\{K_{\lambda}\right\}_{\lambda>1}$ is well-fitting.

(2) $K$ is as in (1). Suppose $A$ is a convex, balanced subset of $Y$, such that $A \subset K^{0}$ and, for every real $\lambda>1, K^{0} \subset \lambda A$. Then the family $\left\{S^{0}: S\right.$ finite, $\left.S \subset A\right\}$ has intersection $K$ and is well-fitting. For, $K^{0}=A^{00}=[\cup S]^{00}$, and therefore $K=K^{00}$ $=[\bigcup S]^{0}=\bigcap S^{0}$; also, for every real $\lambda>1, K^{0} \subset \lambda A=\lambda\left(\bigcup S^{00}\right)$.

(3) Let $\left\{K_{\alpha}\right\}_{\alpha \in I}$ be a family of convex, balanced, $\sigma(X, Y)$-closed sets and let $K=\bigcap_{\alpha \in I} K_{\alpha}$. Suppose 0 is a $\tau(Y, X)$-interior point of co $\left(\bigcup_{\alpha \in I} K_{a}^{0}\right)$. Then the family $\left\{K_{\alpha}\right\}_{\alpha \in I}$ is well-fitting. This follows from the fact that the $\tau(Y, X)$-closure of co $\left(\bigcup_{\alpha \in I} K_{\alpha}^{0}\right)$ is $K^{0}$ and consequently, for every $y \in K^{0}$, for every $\beta<1, \beta y$ is a $\tau(Y, X)$ interior point of co $\left(\bigcup_{\alpha \in I} K_{\alpha}^{0}\right)$. (See [10, p. 38]).

(4) Let $\left\{K_{\alpha}\right\}_{\alpha \in I}$ be a finite family of convex, balanced, $\sigma(X, Y)$-closed subsets of $X$, such that for each $\alpha \in I, \tau(X, Y)-\operatorname{Int}\left(K_{\alpha}\right) \neq \varnothing$. Then the family $\left\{K_{\alpha}\right\}_{\alpha \in I}$ is wellfitting. In fact, each $K_{\alpha}^{0}$ is $\sigma(Y, X)$-compact by the Alaoglu-Bourbaki Theorem $\left[10\right.$, p. 84]. Since the family is finite, co $\left(\bigcup_{\alpha \in I} K_{\alpha}^{0}\right)$ is $\sigma(Y, X)$-compact [10, p. 66], and therefore $\operatorname{co}\left(\bigcup_{\alpha \in I} K_{\alpha}^{0}\right)=K^{0}$.

Lemma 3.1. Let $(X, Y),\left(X_{1}, Y_{1}\right)$ be two dual systems of vector spaces. Let $u: X \rightarrow X_{1}$ be a weakly continuous linear map. Let $\left\{K_{\alpha}\right\}_{\alpha \in I}$ be a well-fitting family of convex, balanced, $\sigma(X, Y)$-closed subsets of $X$, directed downward by inclusion, and let $K=\bigcap_{\alpha \in I} K_{\alpha}$.

Then

$$
\operatorname{cl}(u(K))=\bigcap_{\alpha \in I} \operatorname{cl}\left(u\left(K_{\alpha}\right)\right)
$$

Proof. The following identities are justified by the bipolar theorem and basic facts about polars and transposes:

$$
\begin{aligned}
\bigcap_{\alpha \in I} \operatorname{cl}\left(u\left(K_{\alpha}\right)\right) & =\bigcap_{\alpha \in I}\left[u\left(K_{\alpha}\right)\right]^{00}=\bigcap_{\alpha \in I}\left[{ }^{t} u^{-1}\left(K_{\alpha}^{0}\right)\right]^{0} \\
& =\left[\bigcup_{\alpha \in I}{ }^{t} u^{-1}\left(K_{\alpha}^{0}\right)\right]^{0}=\left[{ }^{t} u^{-1}\left(\bigcup_{\alpha \in I} K_{\alpha}^{0}\right)\right]^{0} .
\end{aligned}
$$


By assumption, for every $\lambda>1, K^{0} \subset \lambda\left(\bigcup_{\alpha \in I} K_{\alpha}^{0}\right)$. Therefore

$$
\left[{ }^{t} u^{-1}\left(\bigcup_{\alpha \in I} K_{\alpha}^{0}\right)\right]^{0} \subset\left[\lambda^{-1}{ }^{t} u^{-1}\left(K^{0}\right)\right]^{0}=\lambda[u(K)]^{00}=\lambda \operatorname{cl}(u(K)), \quad \lambda>1 .
$$

Therefore

$$
\bigcap_{\alpha \in I} \operatorname{cl}\left(u\left(K_{\alpha}\right)\right) \subset \bigcap_{\lambda>1} \lambda \operatorname{cl}(u(K))=\operatorname{cl}(u(K)) .
$$

Since the opposite inclusion is trivial, the desired equality follows.

\section{Concentration properties.}

Definition 4.1. Let $(X, Y)$ be a dual system. Let $\mu$ be a cylinder measure on $\mathscr{C}(X, Y)$. The set function $\mu^{*}$ is defined by

$$
\mu^{*}(A)=\inf \{\mu(C): C \in \mathscr{C}(X, Y), C \supset A\} \text { for any } A \subset X .
$$

Definition 4.2. The cylinder measure $\mu$ is said to be concentrated up to $\varepsilon$ on $A$ if $\mu^{*}(A) \geqq 1-\varepsilon$.

Let $\mathscr{A}$ be a family of subsets of $X$. $\mu$ is said to be concentrated on $\mathscr{A}$ if, for every $\varepsilon>0$, there exists $A \in \mathscr{A}$, such that $\mu$ is concentrated up to $\varepsilon$ on $A$.

We show next that, in a rather general geometric situation, concentration up to $\varepsilon$ of a cylinder measure on the sets of a family directed downward by inclusion implies concentration up to $\varepsilon$ on the image of the intersection of the family by any homothecy of scalar $>1$.

In fact, one can prove the slightly stronger result:

THEOREM 4.1. Let $(X, Y)$ be a dual system. Let $\left\{K_{\alpha}\right\}_{\alpha \in I}$ be a well-fitting family of convex, balanced, $\sigma(X, Y)$-closed subsets of $X$, directed downward by inclusion. Let $K=\bigcap_{\alpha \in I} K_{\alpha}$. Let $\mu$ be a cylinder measure on $\mathscr{C}(X, Y)$. Let $\varepsilon \geqq 0$.

The following conditions are equivalent:

(a) For every $\alpha \in I$ and for every finite set $S \subset K_{\alpha}^{0}, \mu\left(S^{0}\right) \geqq 1-\varepsilon$.

(b) For every convex, balanced, $\sigma(X, Y)$-closed cylinder set $C \supset K, \mu(C) \geqq 1-\varepsilon$.

(c) For every $\lambda>1, \mu^{*}(\lambda K) \geqq 1-\varepsilon$.

Proof. (b) $\Rightarrow$ (a) is obvious.

(c) $\Rightarrow($ b). Suppose $C \supset K$, where $C$ is convex, balanced, $\sigma(X, Y)$-closed and $C \in \mathscr{C}(X, Y)$. Then, for every $\lambda>1, \lambda C \supset \lambda K$ and by (c) we have $\mu(\lambda C) \geqq 1-\varepsilon$. Let $\lambda_{n} \downarrow 1$. Then $\lambda_{n} C \downarrow C$, and the partial $\sigma$-additivity of $\mu$ implies $\mu\left(\lambda_{n} C\right) \downarrow \mu(C)$. Thus $\mu(C) \geqq 1-\varepsilon$.

(a) $\Rightarrow$ (c). Suppose $C \supset \lambda K$, with $\lambda>1$. One can write $C=\pi^{-1}(B), B$ Borel in $R^{p}$, $\pi: X \rightarrow R^{p}$ linear and $\sigma(X, Y)$-continuous. Then $C \supset \pi^{-1}(\pi(\lambda K))$. The set $\pi(K)$ is convex and balanced. By a geometric property of the closure of a convex set with nonempty interior (see [10, p. 38]), applied in the subspace spanned by $\pi(K)$ in $R^{p}$, one has

$$
\pi(\lambda K)=\lambda \pi(K) \supset \operatorname{cl}(\pi(K))
$$


Let $A=\bigcup_{\alpha \in I} K_{\alpha}^{0}$. By the well-fitting assumption and Example (2) following Definition 3.1, the family $\left\{S^{0}: S\right.$ finite, $\left.S \subset A\right\}$ is well-fitting and has intersection $K$. Applying Lemma 3.1, we obtain

$$
\operatorname{cl}(\pi(K))=\bigcap\left\{\operatorname{cl}\left(\pi\left(S^{0}\right)\right): S \text { finite, } S \subset K^{0}\right\} .
$$

Since $\mu \circ \pi^{-1}$ is a regular Borel measure in $R^{p}$, one has

$$
\mu \circ \pi^{-1}(\operatorname{cl}(\pi(K)))=\lim _{S} \mu \circ \pi^{-1}\left(\operatorname{cl}\left(\pi\left(S^{0}\right)\right)\right) .
$$

If $S$ is a finite subset of $A$, the fact that $\left\{K_{\alpha}^{0}\right\}_{\alpha \in I}$ is directed upward by inclusion implies that, for some $\beta \in I, S \subset K_{\beta}^{0}$. By (a), $\mu\left(S^{0}\right) \geqq 1-\varepsilon$. But $\pi^{-1}\left(\operatorname{cl}\left(\pi\left(S^{0}\right)\right)\right)$ $\supset \pi^{-1}\left(\pi\left(S^{0}\right)\right) \supset S^{0}$. Hence $\mu \circ \pi^{-1}\left(\operatorname{cl}\left(\pi\left(S^{0}\right)\right)\right) \geqq \mu\left(S^{0}\right) \geqq 1-\varepsilon$, and (1) implies $\mu \circ \pi^{-1}(\mathrm{cl}(\pi(K))) \geqq 1-\varepsilon$.

Since $C \supset \pi^{-1}(\pi(\lambda K)) \supset \pi^{-1}(\operatorname{cl}(\pi(K)))$, this in turn implies $\mu(C) \geqq 1-\varepsilon$.

RemarK. In Theorem 4.1, suppose $K$ is $\sigma(X, Y)$-compact. Then (b) and (c) can be replaced by the condition $\mu^{*}(K) \geqq 1-\varepsilon$. In fact, let $C \in \mathscr{C}(X, Y), C \supset K$. One can write $C=\pi^{-1}(B)$, as in the proof of Theorem 4.1. Then $C \supset \pi^{-1}(\pi(K)) \supset K$. But $\pi(K)$ is compact, hence closed; therefore $\pi^{-1}(\pi(K))$ is convex, balanced and $\sigma(X, Y)$-closed. By $(\mathrm{b}), \mu\left(\pi^{-1}(\pi(K))\right) \geqq 1-\varepsilon$, and consequently $\mu(C) \geqq 1-\varepsilon$.

In general, one cannot replace (c) by the stronger result $\mu^{*}(K) \geqq 1-\varepsilon$. We will show this by means of a general example, inspired in an example of Laurent Schwartz [12].

The following lemma is stated for easy reference. Its content is alluded to in [2, p. 4].

Lemma 4.1. Let $X, Y, Z$ be vector spaces. Suppose

(1) $X \subset Y^{*}$ and $Z \subset Y^{*}$.

(2) Both $X$ and $Z$ separate points of $Y$.

Then

(a) For every $C \in \mathscr{C}(X, Y)$, there is a unique $\psi(C) \in \mathscr{C}(Z, Y)$ such that $C=(\pi \mid X)^{-1}(B)$ implies $\psi(C)=(\pi \mid Z)^{-1}(B)$, where $\pi=\left(y_{1}, \ldots, y_{n}\right): Y^{*} \rightarrow R^{n}$, $y_{1}, \ldots, y_{n} \in Y$ and $B$ is a Borel set in $R^{n}$. The map $\psi$ is a bijection from $\mathscr{C}(X, Y)$ onto $\mathscr{C}(Z, Y)$ and preserves finite set operations. Finally, for every finite set $F \subset Y$, $\psi(\mathscr{S}(X, F))=\mathscr{S}(Z, F)$ and $\psi \mid \mathscr{S}(X, F)$ preserves countable set operations.

(b) For every cylinder measure $\mu$ on $\mathscr{C}(Z, Y)$, the set function $\hat{\psi}(\mu)$ defined by $\hat{\psi}(\mu)(C)=\mu(\psi(C))$ is a cylinder measure on $\mathscr{C}(X, Y)$. The map $\hat{\psi}$ is a bijection from the space of cylinder measures on $\mathscr{C}(Z, Y)$ onto the space of cylinder measures un $\mathscr{C}(X, Y)$.

EXAmPLE. Let $X$ be a nonreflexive Banach space. For $r \geqq 0$, let

$$
B_{r}=\{x \in X:\|x\| \leqq r\} .
$$

Suppose one can choose $y_{0} \in X^{\prime}$ such that $\left\|y_{0}\right\|=1$ and $y_{0}\left(B_{1}\right)=(-1,1)$. Choose then $z_{0} \in X^{\prime \prime}$ such that $\left\|z_{0}\right\|=1$ and $z_{0}\left(y_{0}\right)=1 . z_{0}$ exists by the Hahn-Banach theorem and necessarily $z_{0} \notin X$. 
The set function $\delta_{z_{0}}=$ unit point mass at $z_{0}$ is a cylinder measure on $\mathscr{C}\left(X^{\prime \prime}, X^{\prime}\right)$. (In fact, it is a regular Borel measure on $X^{\prime \prime}$.) In the context of Lemma 4.1, put $Y=X^{\prime}, Z=X^{\prime \prime}$, and let $\nu=\hat{\psi}\left(\delta_{z_{0}}\right)$. We want to show

(a) For every $r>1, \nu^{*}\left(B_{r}\right)=1$,

(b) $\nu^{*}\left(B_{1}\right)=0$; in fact $\nu\left(y_{0}^{-1}\left(y_{0}\left(B_{1}\right)\right)\right)=0$.

(a) Let $r>1$. Suppose $C \in \mathscr{C}(X, Y), C \supset B_{r}$. Then there exist $y_{1}, \ldots, y_{n} \in Y$ and a Borel set $B$ in $R^{n}$ such that $C=\pi_{X}^{-1}(B)$. (We are using here the terminology of Lemma 4.1(a), with $\pi_{X}=\pi \mid X$.) Since $B_{r}=r B_{1}$, one can proceed as in the proof of Theorem 4.1 to show

$$
C \supset \pi_{X}^{-1}\left(\operatorname{cl}\left(\pi_{X}\left(B_{1}\right)\right)\right) \text {. }
$$

Now $\nu\left(\pi_{\bar{X}}^{-1}\left(\mathrm{cl}\left(\pi_{X}\left(B_{1}\right)\right)\right)\right)=\delta_{z_{0}}\left(\pi_{X^{\prime \prime}}^{-1}\left(\operatorname{cl}\left(\pi_{X}\left(B_{1}\right)\right)\right)\right)$. Let $B_{\rho}^{\prime \prime}=\left\{z \in X^{\prime \prime}:\|z\| \leqq \rho\right\}$ for $\rho \geqq 0$. Since $B_{1}$ is $\sigma\left(X^{\prime \prime}, X^{\prime}\right)$-dense in $B_{1}^{\prime \prime}$ and $B_{1}^{\prime \prime}$ is $\sigma\left(X^{\prime \prime}, X^{\prime}\right)$-compact and $\pi_{X^{\prime \prime}}$ is $\sigma\left(X^{\prime \prime}, X^{\prime}\right)$-continuous, it follows that $\mathrm{cl}\left(\pi_{X}\left(B_{1}\right)\right)=\pi_{X^{\prime \prime}}\left(B_{1}^{\prime \prime}\right)$. Therefore

$$
\delta_{z_{0}}\left(\pi_{X^{\prime \prime}}^{-1}\left(\mathrm{cl}\left(\pi_{X}\left(B_{1}\right)\right)\right)\right)=\delta_{z_{0}}\left(\pi_{X^{\prime \prime}}^{-1}\left(\pi_{X^{\prime \prime}}\left(B_{1}^{\prime \prime}\right)\right)\right)=1,
$$

because $z_{0} \in B_{1}^{\prime \prime}$. Hence $\nu(C)=1$ and $\nu^{*}\left(B_{r}\right)=1$.

(b) On the other hand, $\nu\left(y_{0}^{-1}\left(y_{0}\left(B_{1}\right)\right)\right)=\nu\left(y_{0}^{-1}(-1,1)\right)=\delta_{z_{0}}\left(\left\{z \in X^{\prime \prime}:\left|z\left(y_{0}\right)\right|<1\right\}\right)$ $=0$ because $z_{0}\left(y_{0}\right)=1$.

The following is a concrete realization of the situation described above. Let $X=C_{0}\left(R^{1}\right)=$ space of continuous functions on $R^{1}$, vanishing at $\infty$, with the supremum norm. Then $X^{\prime}=$ space of finite signed measures on $R^{1}$. Let $\gamma$ be the standard Gaussian measure on $R^{1}$, that is, $\gamma(B)=\int_{B}(1 / \sqrt{ }(2 \pi)) \exp \left(-x^{2} / 2\right) d x$, for $B$ Borel in $R^{1}$. Then $\gamma \in X^{\prime}$ and $\|\gamma\|=1$. It is clear that if $f \in X,\|f\| \leqq 1$, then $|\gamma(f)|$ $=\left|\int f d \gamma\right|<\int 1 \cdot d \gamma=1$; hence $\gamma\left(B_{1}\right)=(-1,1)$. Let $g \in X^{\prime \prime}$ be the function on $R^{1}$ identically equal to 1 . We choose $y_{0}=\gamma, z_{0}=g$.

A different concrete example is given by L. Schwartz [12].

Our next proposition deals with the situation in which the family of sets is not necessarily directed.

THEOREM 4.2. Let $(X, Y)$ be a dual system. Let $\left\{K_{\alpha}\right\}_{\alpha \in I}$ be a well-fitting family of convex, balanced, $\sigma(X, Y)$-closed subsets of $X$. Let $K=\bigcap_{\alpha \in I} K_{\alpha}$. Let $\mu$ be a cylinder measure on $\mathscr{C}(X, Y)$. For each $\alpha$, let $\delta_{\alpha}$ be a nonnegative number, and let $\varepsilon=\sum_{\alpha \in I} \delta_{\alpha}$. Suppose, for every $\alpha \in I$ and for every finite set $S \subset K_{\alpha}^{0}, \mu\left(S^{0}\right) \geqq 1-\delta_{\alpha}$.

Then, for every $\lambda>1, \mu^{*}(\lambda K) \geqq 1-\varepsilon$.

Proof. Let $A=\operatorname{co} \bigcup_{\alpha \in I} K_{\alpha}^{0}$. By the well-fitting assumption and Example (2) following Definition 3.1, the family $\left\{S^{0}: S\right.$ finite, $\left.S \subset A\right\}$ is well-fitting and has intersection $K$. By Theorem 4.1, it is enough to prove that, for every finite subset $S$ of $A, \mu\left(S^{0}\right) \geqq 1-\varepsilon$.

Let $S \subset A, S$ finite. Then there exists a finite set of indices $J \subset I$, and finite sets $F_{j} \subset K_{j}^{0}, j \in J$, such that $S \subset\left[\bigcup_{j \in J} F_{j}\right]^{00}$. But this implies $S^{0} \supset\left[\bigcup_{j \in J} F_{j}\right]^{000}$, or $S^{0}$ $\supset \bigcap_{j \in J} F_{j}^{0}$. 
Since $F_{j} \subset K_{j}^{0}, \mu\left(F_{j}^{0}\right) \geqq 1-\delta_{j}$. Therefore $\mu\left(S^{0}\right) \geqq \mu\left(\bigcap_{j \in J} F_{j}^{0}\right) \geqq 1-\left(\sum_{j \in J} \delta_{j}\right) \geqq 1-\varepsilon$. The well-fitting assumption in Theorems 4.1 and 4.2 may be dropped if one requires the cylinder measure $\mu$ to satisfy a mild continuity condition.

We study this situation in Theorems 4.3 and 4.4.

Definition (L. Schwartz [11], [12]). Let $(X, Y)$ be a dual system. Let $A \subset X$, $\varepsilon \geqq 0$. A cylinder measure $\mu$ on $\mathscr{C}(X, Y)$ is said to be scalarly concentrated up to $\varepsilon$ on $A$, if for every $y \in Y,\left(\mu \circ y^{-1}\right)^{*}(y(A)) \geqq 1-\varepsilon$. Let $\mathscr{A}$ be a family of subsets of $X$. $\mu$ is said to be scalarly concentrated on $\mathscr{A}$, if for every $\varepsilon>0$, there exists $A \in \mathscr{A}$ such that $\mu$ is scalarly concentrated up to $\varepsilon$ on $A$.

THeOReM 4.3. Let $(X, Y)$ be a dual system. Let $\left\{K_{\alpha}\right\}_{\alpha \in I}$ be a family of convex, balanced, $\sigma(X, Y)$-closed subsets of $X$, directed downward by inclusion, and such that $K=\bigcap_{\alpha \in I} K_{\alpha}$. Let $\mu$ be a cylinder measure on $\mathscr{C}(X, Y)$, scalarly concentrated on the convex, balanced, $\sigma(X, Y)$-compact subsets of $X$. Let $\varepsilon \geqq 0$.

The following conditions are equivalent:

(a) For every $\alpha \in I$ and for every finite set $S \subset K_{\alpha}^{0}, \mu\left(S^{0}\right) \geqq 1-\varepsilon$.

(b) For every convex, balanced, $\sigma(X, Y)$-closed cylinder set $C \supset K, \mu(C) \geqq 1-\varepsilon$.

(c) For every $\lambda>1, \mu^{*}(\lambda K) \geqq 1-\varepsilon$.

Proof. We only have to show (a) $\Rightarrow$ (c). By Theorem 4.1, it is enough to show that, for every finite set $T \subset K^{0}, \mu\left(T^{0}\right) \geqq 1-\varepsilon$.

Let $A=\bigcup_{\alpha \in I} K_{\alpha}^{0}$. Then $A$ is convex and balanced, because the family is directed upward by inclusion. Therefore $K^{0}=A^{00}=\bar{A}$, the closure being taken with respect to $\tau(Y, X)$.

Let $S$ be finite, $S \subset A$. Then for some $\beta \in I, S \subset K_{\beta}^{0}$, and consequently $\mu\left(S^{0}\right)$ $\geqq 1-\varepsilon$. Now let $T$ be a finite set in $K^{0}, T=\left\{y_{1}, \ldots, y_{m}\right\}$. One can find nets $\left\{y_{j}^{(d)}\right\}_{d \in D}$, such that $y_{j}^{(d)} \in A$ and $y_{j}^{(d)} \rightarrow y_{j}$ in the Mackey topology, for $j=1, \ldots, m$. Let $\pi=\left(y_{1}, \ldots, y_{m}\right), \pi_{(d)}=\left(y_{1}^{(d)}, \ldots, y_{m}^{(d)}\right)$. The scalar concentration assumption on $\mu$ implies $\mu \circ \pi_{(d)}^{-1} \rightarrow \mu \circ \pi^{-1}$ in the weak topology of the space of probability measures on $R^{m}$. (See [12]; also [13, Exposé No. 2].)

Let $Q=\left\{\left(t_{1}, \ldots, t_{m}\right) \in R^{m}: \sup _{1 \leqq j \leqq m}\left|t_{j}\right| \leqq 1\right\}$. Then

$$
\begin{aligned}
\mu\left(T^{0}\right) & =\mu \circ \pi^{-1}(Q) \\
& \geqq \lim _{d \in D} \sup \mu \circ \pi_{(d)}^{-1}(Q) \\
& =\lim _{d \in D} \sup \mu\left(S_{(d)}^{0}\right) \geqq 1-\varepsilon,
\end{aligned}
$$

where $S_{(d)}=\left\{y_{1}^{(d)}, \ldots, y_{m}^{(d)}\right\}$.

The following example shows that the well-fitting hypothesis on the family $\left\{K_{\alpha}\right\}_{\alpha \in I}$ in Theorem 4.1 and the scalar concentration hypothesis on the cylinder measure $\mu$ in Theorem 4.3 are not superfluous.

EXAMPLE. Let $\nu$ be the cylinder measure constructed in the example following Theorem 4.1. Let $F=\operatorname{span}\left\{z_{0}\right\}$, let $F^{\perp}=\left\{y \in X^{\prime}:\langle z, y\rangle=0\right.$ for all $\left.z \in F\right\}$.

Consider the following family of closed subspaces of $X$, of finite codimension: $\left\{S^{\perp} \cap X: S\right.$ finite, $\left.S \subset F^{\perp}\right\}$. Then $\bigcap_{S}\left(S^{\perp} \cap X\right)=\left(\cap_{S} S^{\perp}\right) \cap X=F \cap X=\{0\}$. 
Clearly for all $\lambda \in R^{1}, \nu^{*}(\lambda\{0\})=\nu^{*}(\{0\}) \leqq \nu\left(y_{0}^{-1}\left(y_{0}(0)\right)\right)=0$. On the other hand, $\nu\left(S^{\perp} \cap X\right)=\delta_{z_{0}}\left(S^{\perp}\right)=1$ for all $S$ finite, $S \subset F^{\perp}$, because $z_{0} \in S^{\perp}$.

THEOREM 4.4. Let $(X, Y)$ be a dual system. Let $\left\{K_{\alpha}\right\}_{\alpha \in I}$ be a family of convex, balanced, $\sigma(X, Y)$-closed subsets of $X$. Let $K=\bigcap_{\alpha \in I} K_{\alpha}$. For each $\alpha$, let $\delta_{\alpha}$ be a nonnegative number, and let $\varepsilon=\sum_{\alpha \in I} \delta_{\alpha}$. Let $\mu$ be a cylinder measure on $\mathscr{C}(X, Y)$, scalarly concentrated on the convex, balanced, $\sigma(X, Y)$-compact subsets of $X$. Suppose, for every $\alpha \in I$ and for every finite set $S \subset K_{\alpha}^{0}, \mu\left(S^{0}\right) \geqq 1-\delta_{\alpha}$.

Then, for every $\lambda>1, \mu^{*}(\lambda K) \geqq 1-\varepsilon$.

Proof. Let $A=\operatorname{co} \bigcup_{\alpha \in I} K_{\alpha}^{0}$. Exactly as in the proof of Theorem 4.2, one can show that, for every finite set $S \subset A, \mu\left(S^{0}\right) \geqq 1-\varepsilon$. Next one can use the assumption on $\mu$ to pass to finite subsets of $K^{0}$, as in the proof of Theorem 4.3.

\section{Extension theorems.}

THEOREM 5.1. Let $(X, \mathscr{T})$ be a locally convex Hausdorff topological vector space. Let $\mu$ be a cylinder measure on $\mathscr{C}\left(X, X^{\prime}\right)$.

Then $\mu$ extends to a $\mathscr{T}$-regular Borel measure on $X$, concentrated on the convex, balanced, $\mathscr{T}$-compact subsets of $X$, if and only if the following condition holds:

For every $\varepsilon>0$, there exists a well-fitting family $\left\{K_{\alpha}\right\}_{\alpha \in I}$ of convex, balanced, $\sigma\left(X, X^{\prime}\right)$-closed subsets of $X$, directed downward by inclusion, and such that

(1) $K=\bigcap_{\alpha \in I} K_{\alpha}$ is $\mathscr{T}$-compact,

(2) for every $\alpha \in I$ and for every finite $S \subset K_{\alpha}^{0}, \mu\left(S^{0}\right) \geqq 1-\varepsilon$.

Proof. Let $\varepsilon>0$. Let $\left\{K_{\alpha}\right\}_{\alpha \in I}$ correspond to $\varepsilon$. By Theorem 4.1 (and the remark following it) we have $\mu^{*}(K) \geqq 1-\varepsilon$. The statement follows now applying Prohorov's theorem.

TheOREM 5.2. Let $(X, \mathscr{T})$ be a locally convex Hausdorff topological vector space. Let $\mu$ be a cylinder measure on $\mathscr{C}\left(X, X^{\prime}\right)$, scalarly concentrated on the convex, balanced, $\sigma\left(X, X^{\prime}\right)$-compact subsets of $X$.

Then $\mu$ extends to a $\mathscr{T}$-regular Borel measure on $X$, concentrated on the convex, balanced, $\mathscr{T}$-compact subsets of $X$, if and only if the following condition holds:

For every $\varepsilon>0$, there exists a family $\left\{K_{\alpha}\right\}_{\alpha \in I}$ of convex, balanced, $\sigma\left(X, X^{\prime}\right)$-closed subsets of $X$, directed downward by inclusion, and such that

(1) $K=\bigcap_{\alpha \in I} K_{\alpha}$ is $\mathscr{T}$-compact,

(2) for every $\alpha \in I$ and for every finite $S \subset K_{\alpha}^{0}, \mu\left(S^{0}\right) \geqq 1-\varepsilon$.

Proof. Apply Theorem 4.2 and Prohorov's theorem.

As a consequence of Theorem 5.1, we obtain

TheOREM 5.3 (Le CAM [6], [7]). Let $(X, Y)$ be a dual system. Let $\mu$ be a cylinder measure on $\mathscr{C}(X, Y)$.

Then $\mu$ extends to a $\sigma(X, Y)$-regular Borel measure on $X$, concentrated on the convex, balanced, $\sigma(X, Y)$-compact subsets of $X$, if and only if the following condition holds: 
For every $\varepsilon>0$, there exists a convex, balanced, $\tau(Y, X)$-neighborhood of $0, V$, such that for every finite $S \subset V, \mu\left(S^{0}\right) \geqq 1-\varepsilon$.

Proof. Let $\mathscr{T}=\sigma(X, Y)$; then $(X, \mathscr{T})^{\prime}=Y$. Given $\varepsilon>0$, choose an appropriate $V$. Then $K=V^{0}$ is $\sigma(X, Y)$-compact by the Alaoglu-Bourbaki Theorem [10, p. 84]. Since $\bar{V}=V^{00}=K^{0}$ and $0 \in \tau(Y, X)-\operatorname{Int}(V)$, the family $\left\{S^{0}: S\right.$ finite, $\left.S \subset V\right\}$ is well-fitting. The conclusion follows now from Theorem 5.1.

REMARK. The original statement of this theorem required that $V$ be closed, which is not necessary.

Our next theorem generalizes in a certain sense Theorem 2 of [3]. Completeness plays a crucial role here.

THEOREM 5.4. Let $X$ be a complete Hausdorff locally convex topological vector space. Let $\mathscr{U}$ be a fundamental system of convex, balanced, closed neighborhoods of 0. Let $\mu$ be a cylinder measure on $\mathscr{C}\left(X, X^{\prime}\right)$. Then $\mu$ extends to a regular Borel measure on $X$, concentrated on the convex, balanced, compact subsets of $X$, if and only if the following condition holds:

For every $\varepsilon>0$, there exists a family $\left\{K_{V}: V \in \mathscr{U}\right\}$ of compact convex, balanced subsets of $X$, such that for every finite subset $\mathscr{F} \subset \mathscr{U}, \mu^{*}\left(\bigcap_{V \in \mathscr{F}}\left(K_{V}+V\right)\right) \geqq 1-\varepsilon$.

In order to prove this result, we need

LEMMA 5.1. Let $X$ be a complete Hausdorff topological vector space. Let $\mathscr{U}$ be a fundamental system of closed, symmetric neighborhoods of 0 . For each $V \in \mathscr{U}$, let $K_{V}$ be a compact set, and let $K=\bigcap_{V \in \mathscr{U}}\left(K_{V}+V\right)$.

Then

(a) $K$ is compact.

(b) For every 0-neighborhood $U$, there exists a finite set $\mathscr{F} \subset \mathscr{U}$, such that $K+U$ $\supset \bigcap_{V \in \mathscr{F}}\left(K_{V}+V\right)$.

Proof. (a) Since $X$ is complete, it is enough to show that $K$ is closed and totally bounded. $K$ is closed because $\left(K_{V}+V\right)$ is closed for each $V \in \mathscr{U}$. Given a 0 -neighborhood $W$, choose $V \in \mathscr{U}$ such that $V+V \subset W$. Then choose a finite set $S \subset K_{V}$ such that $K_{V} \subset S+V$. It follows that $K \subset K_{V}+V \subset(S+V)+V \subset S+W$. Therefore $K$ is totally bounded.

(b) Suppose the assertion is false, and let $\mathscr{J}$ be the family of all finite subsets of $\mathscr{U}$. Then for every $J \in \mathscr{J}$, there exists $z(J) \in \bigcap_{V \in J}\left(K_{V}+V\right)$, but $z(J) \notin K+U$. Let $V \in \mathscr{U}$. Then for every $J$ such that $V \in J$, one can write $z(J)=x_{V}(J)+w_{V}(J)$, with $x_{V}(J) \in K_{V}$ and $w_{V}(J) \in V$. We define a net $\{\Phi(J)\}_{J \in \mathscr{I}}$ in $\prod_{V \in \mathscr{U}} K_{V}$ by putting $(\Phi(J))_{V}=x_{V}(J)$ if $V \in J,(\Phi(J))_{V}=p_{V}$ if $V \notin J$, where $p_{V}$ is arbitrarily chosen in $K_{V}$. By Tihonov's theorem $\prod_{V \in \mathscr{U}} K_{V}$ is compact and hence $\Phi$ has a convergent subnet $\{\psi(d)\}_{d \in D}$. Let $x_{V}=\lim _{d}(\Phi(J(d)))_{V}=\lim _{d} x_{V}(J(d))$. Then $x_{V} \in K_{V}$.

We show now that the net $\left\{x_{V}\right\}_{V \in \mathscr{U}}$ is Cauchy. For, let $W$ be a closed neighborhood of 0 . Choose $V \in \mathscr{U}$ such that $V+V \subset W$. Let $V^{\prime} \in \mathscr{U}, V^{\prime \prime} \in \mathscr{U}, V^{\prime} \subset V, V^{\prime \prime} \subset V$. Then for every $J \in \mathscr{J}$ such that $V^{\prime} \in J, V^{\prime \prime} \in J$, one has

$$
x_{V^{\prime}}(J)-x_{V^{\prime \prime}}(J)=w_{V^{\prime \prime}}(J)-w_{V^{\prime}}(J) \in V^{\prime \prime}+V^{\prime} \subset W .
$$


Since eventually $V^{\prime} \in J(d), V^{\prime \prime} \in J(d)$, it follows that

$$
x_{V^{\prime}}-x_{V^{\prime \prime}}=\lim _{d}\left(x_{V^{\prime}}(J(d))-x_{V^{\prime \prime}}(J(d))\right) \in W .
$$

Since $X$ is complete, $\left\{x_{V}\right\}_{V \in \mathscr{U}}$ converges. Let $x_{0}$ be its limit.

We will show now that $x_{0} \in K$. In fact, for any $V \in \mathscr{U}, x_{V}-x_{0} \in \operatorname{cl}\left(V+V^{\prime}\right)$ for every $V^{\prime} \in \mathscr{U}, V^{\prime} \subset V$. But this implies $x_{V}-x_{0} \in V$ and therefore $x_{0} \in x_{V}+V$ $\subset K_{V}+V$. Hence $x_{0} \in K$.

Finally, write $z(J)-x_{0}=\left[x_{V}(J)-x_{V}\right]+\left[x_{V}-x_{0}\right]+w_{V}(J)$. Choose a neighborhood $W$ of 0 such that $W+W+W \subset U$. Then choose $V \in \mathscr{U}$ such that $x_{V}-x_{0} \in W$ and $w_{V}(J) \in W$ (for all $J$ ). Then there exists $d \in D$ such that $x_{V}(J(d))-x_{V} \in W$. This implies $z(J(d))-x_{0} \in U$, or $z(J(d)) \in x_{0}+U \subset K+U$, a contradiction.

Proof of Theorem 5.4. The condition is clearly necessary. For each finite subset $J \subset \mathscr{U}$, call $K_{J}=\bigcap_{V \in J}\left(K_{V}+V\right)$. Then $\left\{K_{J}: J\right.$ finite $\left.\subset \mathscr{U}\right\}$ is a family of convex, balanced, $\sigma\left(X, X^{\prime}\right)$-closed subsets of $X$, directed downward by inclusion. We will show next that the family is well-fitting. Since $K=\bigcap_{J} K_{J}$ is compact by Lemma 5.1(a), the result will follow then from Theorem 5.1.

Since $\bigcup_{J} K_{J}^{0}$ is convex and balanced and $\operatorname{cl}\left(\bigcup_{J} K_{J}^{0}\right)=\left[\bigcup_{J} K_{J}^{0}\right]^{00}=K^{0}$ (the closure is taken in the Mackey topology), in order to prove the well-fitting property it is enough to show that $0 \in \tau\left(X^{\prime}, X\right)-\operatorname{Int}\left(\bigcup_{J} K_{J}^{0}\right)$. Let $U$ be a neighborhood of 0 . By Lemma 5.1(b), there is a $J$ such that $K+U \supset K_{J}$, or $(K+U)^{0} \subset K_{J}^{0}$. It follows that $\bigcup_{U}(K+U)^{0} \subset \bigcup_{J} K_{J}^{0}$. But $(K+U)^{0} \supset\left(\frac{1}{2} K^{0}\right) \cap\left(\frac{1}{2} U^{0}\right)$. Since $\bigcup_{U}\left(\frac{1}{2} U^{0}\right)=X^{\prime}$, we have

$$
\bigcup_{J} K_{J}^{0} \supset \bigcup_{U}\left[\left(\frac{1}{2} K^{0}\right) \cap\left(\frac{1}{2} U^{0}\right)\right]=\frac{1}{2} K^{0} .
$$

But $\frac{1}{2} K^{0}$ is a $\tau\left(X^{\prime}, X\right)$-neighborhood of 0 because the set $K$ is compact, convex and balanced.

As a corollary, we obtain a proposition which essentially contains Theorem 2 and Corollary 2.1 of [3]

TheOREM 5.5. Let $X$ be a Fréchet space. Let $\mathscr{U}=\left\{V_{n}\right\}_{n \in N}$ be a countable fundamental system of convex, balanced, closed neighborhoods of 0 . Let $\mu$ be a cylinder measure on $\mathscr{C}\left(X, X^{\prime}\right)$.

Then $\mu$ extends to a regular Borel measure on $X$ (concentrated on the convex, balanced, compact subsets of $X$ ) if and only if the following condition holds:

For every $\varepsilon>0$, for every $V_{n} \in \mathscr{U}$, there exists a finite-dimensional subspace $F_{n}$ of $X$ such that $\mu^{*}\left(F_{n}+V_{n}\right) \geqq 1-\varepsilon$.

Proof. We begin with some geometric remarks. Let $F$ be a finite-dimensional subspace of $X$, and let $V$ be a convex, balanced, closed neighborhood of 0 . We want to show that there exist a subspace $H \subset F$, a finite subset $T \subset X^{\prime}$, and a number $\beta>0$, such that

(1) for all $r \geqq 0,(F+V) \cap\left(r T^{0}\right) \subset\left[H \cap\left((r+\beta) T^{0}\right)\right]+V$,

(2) for all $r \geqq 0, H \cap\left(r T^{0}\right)$ is compact. 
In fact, let $S=\bigcap_{\lambda>0}(\lambda V)$. Then $S$ is a closed subspace. Let $H$ be a subspace of $F$, such that $(F \cap S) \oplus H=F$. Then $F+V=H+V$ and $H \cap S=\{0\}$. It follows that $\operatorname{cl}\left(H^{\perp}+S^{\perp}\right)=X^{\prime}$, the closure being taken in the $\sigma\left(X^{\prime}, X\right)$-topology. Now $S^{\perp}=S^{0}$ $=\left[\bigcup_{\lambda>0}\left(\lambda V^{0}\right)\right]^{00}=\operatorname{cl}\left(\bigcup_{\lambda>0}\left(\lambda V^{0}\right)\right)$. Let $G=\bigcup_{\lambda>0}\left(\lambda V^{0}\right)$. Then $G$ is a subspace (not necessarily closed) and $\operatorname{cl}\left(H^{\perp}+G\right)=X^{\prime}$. Since $H^{\perp}$ is of finite codimension, one can choose $y_{j} \in G, j=1, \ldots, m$, such that if $T=\left\{y_{1}, \ldots, y_{m}\right\}$, then $\operatorname{span}(T) \oplus H^{\perp}=X^{\prime}$. Since $T \subset G$, there exists $\beta>0$, such that $T \subset \beta V^{0}$. Now suppose $x \in(F+V) \cap\left(r T^{0}\right)=(H+V) \cap\left(r T^{0}\right)$. Then one can write $x=h+v, h \in H, v \in V$. Since $x \in\left(r T^{0}\right)$ and $v \in\left(\beta T^{0}\right)$, it follows that $h \in(r+\beta) T^{0}$. Therefore

$$
x \in\left[H \cap\left((r+\beta) T^{0}\right)\right]+V .
$$

Let $y \in X^{\prime}$. One can write $y=z+\sum_{j=1}^{m} \lambda_{j} y_{j}, z \in H^{\perp}, \lambda_{j} \in R, j=1, \ldots, m$. If $x \in H \cap\left(r T^{0}\right)$, then $\langle x, y\rangle=\sum_{j=1}^{m} \lambda_{j}\left\langle x, z_{j}\right\rangle$ and finally $\sup _{x \in H \cap\left(r T^{0}\right)}|\langle x, y\rangle|$ $\leqq r \sum_{j=1}^{m}\left|\lambda_{j}\right|$. It easily follows that $H \cap\left(r T^{0}\right)$ is bounded (because $H$ is finite dimensional, for example). Since $H \cap\left(r T^{0}\right)$ is closed, it is compact (and also convex and balanced).

Let $\varepsilon>0$. For each $n \in N$, choose a finite-dimensional subspace $F_{n}$, such that $\mu^{*}\left(F_{n}+V_{n}\right) \geqq 1-\varepsilon / 2^{n+1}$. Take $H_{n}, T_{n}, \beta_{n}$ as in the previous paragraph, and choose $r_{n}$ so that $\mu^{*}\left(r_{n} T_{n}^{0}\right) \geqq 1-\varepsilon / 2^{n+1}$. This is possible because of the partial $\sigma$-additivity of $\mu$. Applying now Theorem 4.2 and Example (4) following Definition 3.1, it follows that for every $\lambda>1, \mu^{*}\left(\lambda\left[\left(F_{n}+V_{n}\right) \cap\left(r_{n} T_{n}^{0}\right)\right]\right) \geqq 1-\varepsilon / 2^{n}$. Therefore $\mu^{*}\left(\lambda\left[K_{n}+V_{n}\right]\right) \geqq 1-\varepsilon / 2^{n}$ for every $\lambda>1$, where $K_{n}=\left[H_{n} \cap\left(\left(r_{n}+\beta_{n}\right) T^{0}\right)\right]$.

Let $J \subset N, J$ finite. By the same argument as above, for every $\lambda>1$,

$$
\mu^{*}\left(\bigcap_{n \in J}\left(\lambda K_{n}+\lambda V_{n}\right)\right) \geqq 1-\sum_{n \in J}\left(\varepsilon / 2^{n}\right) \geqq 1-\varepsilon .
$$

The result follows now from Theorem 5.4.

6. Application to $l_{p}$ spaces.

Proposition 6.1. Let $p \geqq 1, X=l_{p}$. For $m<n$, let

$$
C_{\delta}(m, n)=\left\{x=\left(x_{j}\right)_{j \in N}: \sum_{j=m+1}^{n}\left|x_{j}\right|^{p} \leqq \delta^{p}\right\} .
$$

Let $\mu$ be a cylinder measure on $\mathscr{C}\left(X, X^{\prime}\right)$ scalarly concentrated on the $\sigma\left(X, X^{\prime}\right)$ compact, convex, balanced subsets of $X$.

Then $\mu$ extends to a regular Borel measure on $X$ if and only if the following condition holds:

For every $\varepsilon>0$, for every $\delta>0$, there exists $m \in N$ such that $n>m$ implies $\mu\left(C_{\delta}(m, n)\right) \geqq 1-\varepsilon$.

Proof. Let $e_{j}$ be the $j$ th unit coordinate vector and let $F_{m}=\operatorname{span}\left\{e_{1}, \ldots, e_{m}\right\}$. Then

$$
\begin{aligned}
F_{m}^{\delta} & =\left\{x: \text { distance }\left(x, F_{m}\right) \leqq \delta\right\} \\
& =\left\{x: \sum_{j=m+1}^{\infty}\left|x_{j}\right|^{p} \leqq \delta^{p}\right\}=\bigcap_{n=m+1}^{\infty} C_{\delta}(m, n) ;
\end{aligned}
$$


moreover, $\left\{C_{\delta}(m, n)\right\}_{n>m}$ is a decreasing sequence of convex, balanced, $\sigma\left(X, X^{\prime}\right)$ closed cylinder subsets of $X$. Given $\varepsilon>0, \delta>0$, choose an appropriate $m$. By Theorem 4.3 one has, for every $\lambda>1, \mu^{*}\left(\lambda F_{m}^{\delta}\right) \geqq 1-\varepsilon$. The conclusion follows now by applying Theorem 5.5 . (We have proved the sufficiency only; the necessity is clear.)

In the case $p>1$, the weak compactness of the balls permits a further simplification:

Proposition 6.2. Let $p>1$. $X=l_{p}$. For $n \in N$, let

$$
C_{r}^{n}=\left\{x=\left(x_{j}\right)_{j \in N}: \sum_{j=1}^{n}\left|x_{j}\right|^{p} \leqq r^{p}\right\} .
$$

Let $\mu$ be a cylinder measure on $\mathscr{C}\left(X, X^{\prime}\right)$, scalarly concentrated on the balls of $X$. Then $\mu$ extends to a regular Borel measure on $X$ if and only if the following condition holds:

For every $\varepsilon>0$, there exists $r>0$ such that for all $n \in N, \mu\left(C_{r}^{n}\right) \geqq 1-\varepsilon$.

Proof. Similar to the proof of Proposition 6.1, but using Theorem 5.2. (The extension obtained is a regular Borel measure for the weak topology, but since the space is Polish, it is also regular for the norm topology.)

\section{REFERENCES}

1. N. Bourbaki, Intégration, Chap. IX, Hermann, Paris, 1969.

2. R. M. Dudley, Random linear functionals, Trans. Amer. Math. Soc. 136 (1969), 1-24.

3. R. M. Dudley, J. Feldman and L. Le Cam, On seminorms and probabilities and abstract Wiener spaces, Ann. of. Math. (2) 93 (1971), 390-408.

4. X. Fernique, Processus linéaires, processus généralisés, Ann. Inst. Fourier (Grenoble) 17 (1967), fasc. 1, 1-92. MR 36 \#4628.

5. R. K. Getoor, On characteristic functions of Banach space valued random variables, Pacific J. Math. 7 (1957), 885-896. MR 19, 584.

6. L. Le Cam, Remarques sur les variables aléatoires dans les espaces vectoriels non séparables. Le calcul des probabilités et ses applications, Colloq. Internat. du Centre National de la Recherche Scientifique (Paris, 1958), Centre National de la Recherche Scientifique, Paris, 1959, pp. 39-53. MR 21 \#4463.

7. - - Extending cylinder measures, 1970 (unpublished manuscript).

8. E. Mourier, Eléments aléatoires dans un espace de Banach, Ann. Inst. H. Poincaré 13 (1953), 161-244. MR 16, 268.

9. Ju. V. Prohorov, The method of characteristic functionals, Proc. Fourth Berkeley Sympos. Math. Statist. and Prob. (Berkeley, Calif., 1960), vol. 2, Univ. of California Press, Berkeley, Calif., 1961, pp. 403-419. MR 24 \#A3670.

10. H. H. Schaefer, Topological vector spaces, Macmillan, New York, 1966. MR 33 \#1689.

11. L. Schwartz, Extensions du théorème de Sazonov-Minlos à des cas non hilbertiens, C.R. Acad. Sci. Paris Sér. A-B 265 (1967), A832-A834. MR 37 \#2275.

12. - Radon measures on arbitrary topological spaces, Tata Institute, Bombay, India (to appear).

13. A. Badrikian, Séminaire sur les fonctions aléatoires linéaires et les mesures cylindriques, Lecture Notes in Math., no. 139, Springer-Verlag, Berlin and New York, 1970.

Massachusetts Institute of Technology, CAMBridge, MassachusetTS 02139 